\title{
Classificação Automática de Modulações DP m-PSK e DP m-QAM em Receptores Ópticos Coerentes Flexíveis
}

\author{
Antônio M. C. Pereira ${ }^{1}$, Rafael S. Furtado ${ }^{1}$, Diego A. Amoedo ${ }^{2}$, Myke D. M. Valadão ${ }^{1}$, \\ Celso B. Carvalho ${ }^{1}$, André L. A. da Costa $^{3}$, Waldir S. S. Júnior ${ }^{1}$ \\ ${ }^{1}$ Universidade Federal do Amazonas (DTEC/CETELI/UFAM), AM-Brasil \\ ${ }^{2}$ Agência Nacional de Telecomunicações (ANATEL), AM-Brasil \\ ${ }^{3}$ Universidade Federal de Uberlândia (FEELT/UFU), MG-Brasil \\ Emails: \{rafael.furtado1997, myke.medeiros\}@gmail.com, diegoalves@anatel.gov.br, \\ \{antoniopereira, ccarvalho_,waldirjr\}@ufam.edu.br, alacosta@ufu.br
}

Resumo-A buscar por formas de uso eficiente das redes ópticas tornam-se atualmente alternativas à crescente demanda por banda passante e taxas de transmissão. Tecnologias como o flex grid alocam dinamicamente os canais conforme as demandas da rede. Técnicas de classificação automática de modulação fornecem informações que auxiliam na definição de parâmetros de transmissão, como modulações ideais para o meio de transmissão, algoritmos para recuperação da frequência e da fase, transmissão mono ou multi portadoras, algoritmos de correção de erros e o ganho dos amplificadores. Neste artigo apresenta-se uma pesquisa a respeito do uso dos algoritmos random forest, adaboost e árvore de decisão para a classificação automática de modulações em receptores ópticos coerentes flexíveis. Para tal, utilizou-se um setup back-to-back entre um receptor e um transmissor Além disso, gerou-se 93.600 sinais com modulações DP m-PSK e DP m-QAM e treze níveis de ruído. Por fim, os resultados apontam a obtencão de valores de acurácia acima de $97 \%$ para a classificação de modulações utilizando apenas uma das componentes em fase ou em quadratura do sinal com o modelo random forest, demonstrando que a pesquisa é promissora.

Palavras-Chave-Classificação automática de modulação, Aprendizado de máquina, Redes ópticas.

Abstract-The search for efficient ways to make use of the available optical networks are a viable alternative for the ever increasing demand for higher transmission rates. Technologies such as flex grid, can dynamically allocate the channels accordingly with the network demands. Automatic modulation classification techniques provide information that can be used to define some transmission parameters such as the ideal modulation format for the current transmission medium, frequency and phase recovery algorithms, single or multi transmission carriers, error correction algorithms and the amplifiers gain. This paper presents a research about the usage of automatic modulation classificators in flexible optical coherent receivers. To accomplish this, a back-to-back setup between a receiver and transmitter was used. Furthermore, were generated 93,600 signals modulated as DP m-PSK and DP m-QAM, each with thirteen noise levels. Lastly, the results obtained was $97 \%$ accuracy of the modulation classification using one component of the signal, being the quadrature or phase alone, showing that is a promising research.

Keywords-Automatic modulation classification, Machine learning, Optical networks.

Antônio M. C. Pereira, Myke D. M. Valadão, Rafael S. Furtado, Celso B. Carvalho e Waldir S. S. Júnior, Universidade Federal do Amazonas (DTEC/CETELI/UFAM), André L. A. da Costa, Universidade Federal de Uberlândia (UFU), Uberlândia, Diego A. Amoedo, Agência Nacional de Telecomunicações (ANATEL), Manaus-AM. E-mails: \{antoniopereira, ccarvalho_,waldirjr\}@ufam.edu.br, diegoalves@anatel.gov.br, alacosta@ufu.br, \{rafael.furtado1997, myke.medeiros\}@gmail.com

\section{INTRODUÇÃO}

Um estudo realizado por um grande fabricante de dispositivos de redes de telecomunicações, aponta uma projeção de 5,3 bilhões de usuários de Internet em 2023, o que representa $66 \%$ da população mundial [1]. Tomando-se o intervalo de análise, entre 2018 e 2023, tal estudo evidenciou um aumento de $6 \%$ ao ano de novas conexões a Internet [1]. Com a conclusão do processo de padronização da quinta geração das redes móveis em 2020 [2], as aplicações de machine to machine em diversas áreas da indústria possibilitaram um crescimento acelerado da tecnologia em Internet of things (IoT), na qual, pessoas, processos, dados e coisas se conectam à Internet e entre si. A previsão é que a IoT representará o maior número de conexões e será responsável por $50 \%$ dos dispositivos conectados em 2023. Os smartphones por sua vez, representarão o segundo maior número de dispositivos conectados, ou seja, $7 \%$ do total, seguidos por smart TVs, set-top boxes, digital media adapters e gaming consoles [1]. Além das tecnologias relacionadas acima, ainda está prevista a Internet tátil, na qual, a latência não poderá ultrapassar $1 \mathrm{~ms}$, a uma taxa de $10 \mathrm{Gbps}$, possibilitando aplicações como tele cirurgia [3]. Segundo este mesmo fabricante, o número de dispositivos conectados em 2030 será da ordem de 500 bilhões [4], um número significativamente maior do que a população mundial, tornando-se um grande desafio para as instituições de pesquisa e para a indústria. Dessa forma, nos últimos dois anos, iniciaram-se estudos sobre a sexta geração das redes móveis, a qual, promete latências ultrabaixas, taxas da ordem 100 Gbps e áreas de cobertura supostamente onipresentes [5]. Diante deste cenário, tanto as redes ópticas de acesso de rádio (RAN), quanto as redes ópticas de transporte (OTN), surgem como alternativas importantes para a infraestrutura de acesso e núcleo das redes de telecomunicações, capazes de suportar o tráfego que será gerado pelo volume de dispositivos conectados. Em algumas pesquisas, discutem-se novas estratégias para as redes RAN com a finalidade de atender as redes móveis de quinta e sexta gerações [6].

As redes OTN estão em constante evolução, e na última década, a microeletrônica, a fotônica integrada [7], a infraestrutura atualmente instalada e a multiplexação por divisão de comprimento de ondas (WDM), possibilitaram a migração de canais com modulações não coerentes e taxas de $10 \mathrm{Gbps}$, para canais com modulações coerentes mono ou multiportadoras de 100 Gbps, 200 Gbps, 400 Gbps, 800 Gbps e 1,2 Tbps [8], [9]. Esta evolução, por meio do processamento digital de sinais, proporcionou a compensação de forma eletrônica dos efeitos lineares da dispersão cromática (CD) e da dis- 
persão modo de polarização (PMD), além dos efeitos nãolineares relacionados ao coeficiente Kerr [10]. O emprego de amplificadores híbridos, técnicas de processamento de sinais e modulações de formato geométricos por multiplexação por divisão de polarização, possibilitou a demonstração de uma transmissão ao longo de $6300 \mathrm{~km}$ a uma taxa de $74,38 \mathrm{Tbps}$ nas bandas $C$ e $L$ de amplificação óptica [11]. A evolução destas redes OTN também ocorreu nos dispositivos de comutação com o emprego de comutadores seletivos de comprimentos de ondas (WSS) e os comutadores OTN responsáveis pelas conexões cruzadas entre enlaces de longas distância e as redes metropolitanas.

Ainda em 2012, o ITU por meio da recomendação G.694.1 [12], padronizou o espaçamento flexível das redes WDM, otimizando assim o uso do espectro óptico, possibilitando a coexistência de canais com diferentes modulações, taxas e bandas de transmissão. Tal recomendação define que os espaçamento mínimo entre os canais é de $12,5 \mathrm{GHz}$, ao contrário dos $37,5 \mathrm{GHz}$ do padrão anterior.

Nos últimos anos, a inteligência artificial (AI), bem como o aprendizado de máquina (do inglês, machine learning, ML), se consolidaram como áreas de pesquisa amplamente exploradas com o objetivo gerar soluções em diferentes áreas do conhecimento, modificando o estilo de vida, a vida em comunidade, a produtividade, a saúde e a segurança. Assim, no que diz respeito às redes de telecomunicações, as pesquisas relacionadas à AI e ML são aplicáveis nas áreas de transmissão, roteamento e gerenciamento [13]. Nas redes OTN, as técnicas de AI e ML são empregadas nos transponderes ópticos para a caracterização de amplitude e ruído de fase [13], no controle dos amplificadores ópticos [13], na identificação da degradação dos sinais devido aos efeitos lineares de CD e PMD [13], no monitoramento da relação sinal ruído óptica [13], na mitigação dos efeitos não lineares nos receptores [14], [15] e na estimativa da qualidade de transmissão óptica [13]. Além disso, o AI e ML são utilizados nas redes OTN para a classificação automática de modulações [16]-[21], possibilitando o ajuste dinâmico, de tais redes, à qualidade do meio de transmissão, sem a interferência humana, por meio da seleção de taxas de transmissão adequadas ou transmissões em única ou multiportadoras. Desta forma, este trabalho tem por objetivo apresentar um método para classificação automática de modulações em receptores ópticos coerentes flexíveis, utilizando-se para tal, diferentes algoritmos de aprendizado de máquina [22]. Por fim, a organização do artigo é feita da seguinte maneira: na Seção II, apresenta-se trabalhos relacionados que contribuíram para este artigo. $\mathrm{Na}$ Seção III, apresenta-se o sistema proposto, com os algoritmos, projeto dos classificadores, setup e resultados. Por último, na Seção IV, as conclusões.

\section{A. Contribuições do artigo}

Este trabalho apresenta um estudo sobre a utilização dos algoritmos random forest, adaboost e árvore de decisão para a classificação automática de modulações em receptores ópticos coerentes flexíveis. Para extração de características e construção da base de dados, implementou-se um setup de simulação back-to-back, no software VPIphotonics, entre um receptor e um transmissor. Além disso, gerou-se 93.600 sinais com modulações DP m-PSK e DP m-QAM (do inglês, respectivamente, dual-polariozation quadrature phase-shift keying e dual-polariozation quadrature amplitude modulation) e treze níveis de ruído. Os algoritmos utilizados no treinamento e teste para a classificação automática das modulações foram implementados na linguagem de programação Python.

\section{TRABALHOS RELACIONADOS}

Em [19], utiliza-se redes neurais profundas (DNN) para o reconhecimento da modulação em receptores coerentes. A DNN utilizada possui duas camadas ocultas para a extração das características a partir dos histogramas de amplitude, assim os resultados desta etapa servem para a detecção da modulação pela rede. Neste trabalho, foi utilizado um setup de transmissão em laboratório com três diferentes formatos de modulação, sendo o QPSK, 16-QAM e 64-QAM no transmissor. No receptor coerente, os sinais modulados são recebidos e regenerados por um DSP (digital signal processor). Durante a etapa de regeneração, após o bloco de equalização CMA (constant modulus algorithm) são extraídos os histogramas do sinal contendo 80 colunas e por fim classificada a modulação. Foram avaliados 60 sinais, sendo 20 para cada modulação utilizada. A acurácia foi de $100 \%$ para os casos de teste.

Em [18], utiliza-se o algoritmo SVM para classificação do formato de modulação e estimativa da OSNR (optical signalto-noise ratio). Neste caso, usa-se um setup montado em laboratório, que faz uso de um gerador de forma de onda conectado a um laser com cavidade externa, utilizado como transmissor, sendo o ruído adicionado pela inserção de um amplificadores de fibra dopada de Érbio (do inglês, Erbiumdoped fiber amplifier, EDFA) e um acoplador óptico. O sinal de uma saída do acoplador é captado por um fotodiodo, por fim este sinal é utilizado para projeto do classificador. A acurácia ficou entre $87 \%$ e $100 \%$ para a classificação do formato de modulação e erros entre $0,7 \mathrm{~dB}$ e $3,5 \mathrm{~dB}$ para a estimativa do valor de OSNR. Em outro trabalho similar [17], apresentase um algoritmo para classificação da modulação baseando-se na amplitude de um sinal recebido em um sistema coerente. Nesta pesquisa, usa-se a função de distribuição cumulativa (do inglês, cumulative distribution function, CDF), a qual é aplicada aos valores de amplitude normalizada dos sinais. As modulações utilizadas foram 4/8/16/32/64-QAM, através de um sistema back-to-back, transmitido com um valor de OSNR previamente conhecido. A acurácia foi de $100 \%$ de acerto para a classificação das modulações com valores de OSNR abaixo de determinados limiares.

Em [23], é proposto um algoritmo de identificação do formato de modulação e monitoramento de OSNR usando kNN (k-nearest neighbor) para modulação de intensidade e detecção direta (do inglês, intensity modulation and direct detection, IMDD) em sistemas IMDD-OFDM. Os experimentos são conduzidos sobre uma fibra padrão monomodo, onde um sistema IMDD-OFDM é simulado de ponta-a-ponta. Na recepção, são extraídos histogramas de amplitude, e então utilizados como feature para classificação. Conforme com os resultados experimentais, as modulações 4-QAM, 16-QAM, 32-QAM, 64-QAM e 128-QAM são identificadas com acurácia de $100 \%$ com a potência óptica recebida de $-11 \mathrm{dBm}$. A OSNR, por sua vez, é estimada utilizando as informações dos tipos de modulação, com MSE médio de predição de $0,69 \mathrm{~dB}^{2}$.

\section{SISTEMA PROPOSTO PARA CLASSIFICAÇÃO} AUTOMÁTICA DE MODULAÇÃO EM RECEPTORES ÓPTICOS COERENTES

\section{A. Introdução}

Nesta seção apresenta-se alguns aspectos importantes sobre o sistema óptico simulado (Seção III-B) e sobre a geração dos sinais e extração de características (Seção III-C). A seguir, comenta-se sobre os algoritmos de classificação árvore de decisão (Seção III-D), random forest e adaboost (Seção III-E). Em seguida, apresenta-se como é realizado o treinamento dos classificadores (Seção III-F) e por fim, as métricas utilizadas e a análise de resultados resultados (Seção III-G). 


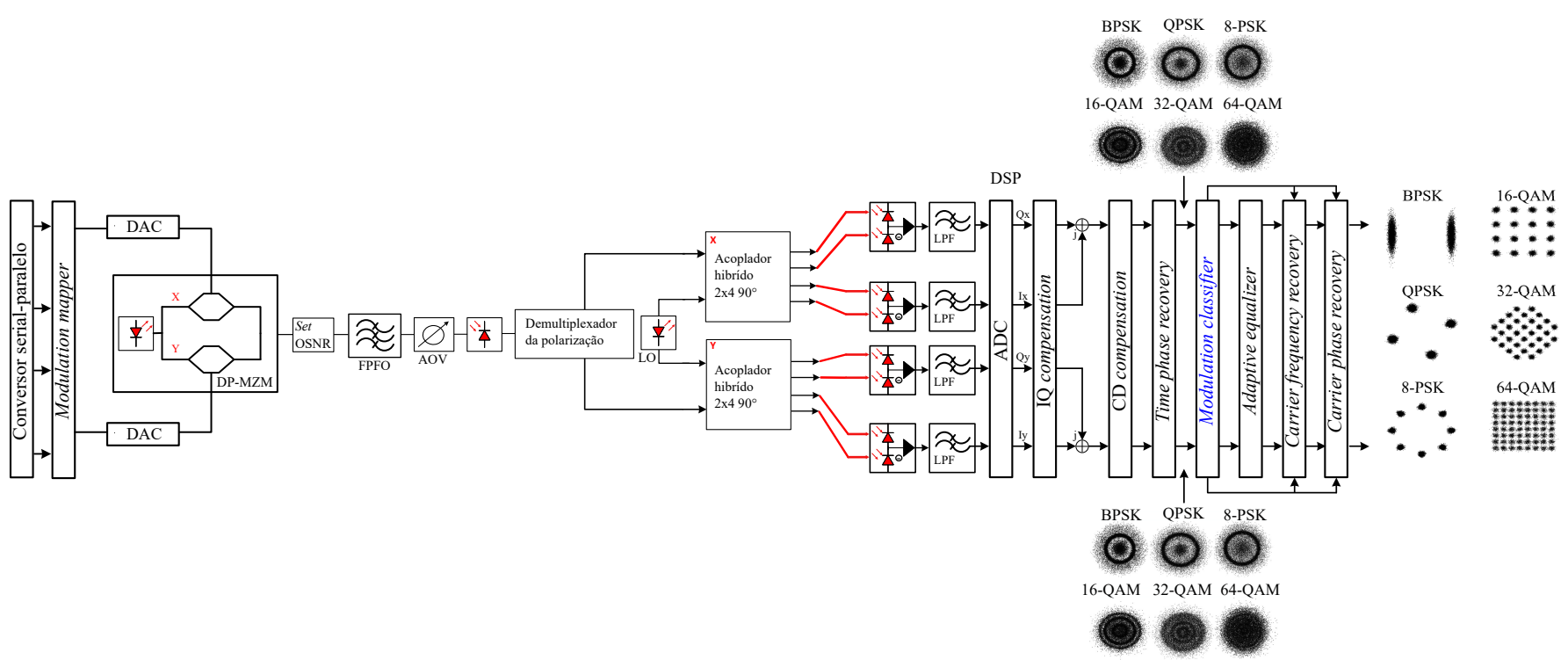

Fig. 1. Diagrama em blocos do transmissor e receptor óptico coerente utilizado na geração dos sinais para treino e teste. Conversor serial-paralelo; DAC: conversor digital-analógico; Diodo laser; DP-MZM: modulador Mach-Zehnder de duplo controle; set OSNR: define o valor da OSNR em dB/0,1 nm; FPFO filtro passa-faixa óptico; AOV: atenuador óptico variável; LO: Oscilador Local; PD: fotodiodo; Receptor óptico coerente; LPF: filtro passa-baixa; ADC: conversor analógico-digital; DSP: processador de sinais digitais.

\section{B. Esquema de simulação}

A Figura 1 ilustra o setup back-to-back de simulação do VPIphotonics, composto no transmissor, por um conversor serial paralelo e um mapeador de modulações. Tais modulações são posteriormente convertidas de sinais digitais para analógicos, os quais, modularão a portadora do laser na frequência $f_{\mathrm{o}}=192,1 \mathrm{THz}$ nos estados de polarizações $\overrightarrow{E_{x}}$ e $\overrightarrow{E_{y}}$. Assim, os sinais modulados são multiplexados, passando inicialmente pelo bloco de ajuste de relação sinal ruído óptica, o qual, apresentará em sua saída um sinal com OSNR fixa em $\mathrm{dB} / 0,1 \mathrm{~nm}$. No receptor, o sinal passará pelo filtro óptico passa faixa (OBPF), centrado em $f_{\mathrm{c}}=192,1 \mathrm{THz}$ e em seguida por um atenuador óptico variável (VOA), com o objetivo de manter a potência no fotodetector constante e igual a $-2 \mathrm{dBm}$. Após o atenuador, os estados de polarizações $\overrightarrow{E_{x}}$ e $\overrightarrow{E_{y}}$ serão demultiplexados e alimentarão os acopladores híbridos $2 \times 4$ de noventa graus, assim como o sinal do laser do oscilador local centrado em $f_{\mathrm{LO}}=192,1 \mathrm{THz}$. Na sequência, os sinais em fase e quadratura de ambos os estado de polarização passarão pelos fotodetectores, amplificadores de corrente e pelo filtro passa baixa (LPF), chegando ao processador digital de sinais (DSP), onde são convertidos de analógicos para digitais. Em seguida a dispersão cromática é compensada pelo bloco CD compensation, bem como o sincronismo pelo bloco time phase recovery. Desta forma, após os estágios anteriores do DSP, os sinais de apenas um dos estados de polarização são utilizado pelo classificador automático de modulações, proposto por este trabalho, que tem como objetivo indicar aos blocos de recuperação da frequência e da fase das portadoras de transmissão, qual é o tipo de modulação, em um determinado momento, para que tais blocos possam utilizar os algoritmos de estimativa de frequência e fase corretos, resultando em sinais corretamente detectados de ambos os estados de polarização, conforme verificado na saída do diagrama em blocos da Figura 1.

\section{Geração de sinais e extração de características}

Os sinais para projeto e execução (treino e teste) utilizados no classificador foram gerados a partir do bloco Modula- tion classifier destacado em azul na Figura 1. Tais sinais apresentam modulações DP-BPSK, DP-QPSK, DP-8PSK, DP16QAM, DP-32QAM e DP-64QAM (do inglês, respectivamente, dual-polariozation quadrature phase-shift keying $\mathrm{e}$ dual-polariozation quadrature amplitude modulation) com a taxa de símbolos de $28 \mathrm{Gbaud} / \mathrm{s}$ e bits por símbolos, respectivamente iguais a $1,2,3,4,5,6$. Após a passagem dos sinais de interesse pelo bloco de ajuste da relação sinal ruído óptica (OSNR) acrescentou-se a cada um deles um nível específico de ruído, resultando em treze níveis de OSNR, variando de 14 até $20 \mathrm{~dB} / 0,1 \mathrm{~nm}$ com intervalos de $0,5 \mathrm{~dB} / 0,1 \mathrm{~nm}$.

A Figura 2 ilustra os sinais mPSK e mQAM de um dos estados de polarização que chegam à entrada do classificador automático de modulação. Após a aquisição dos sinais, tem

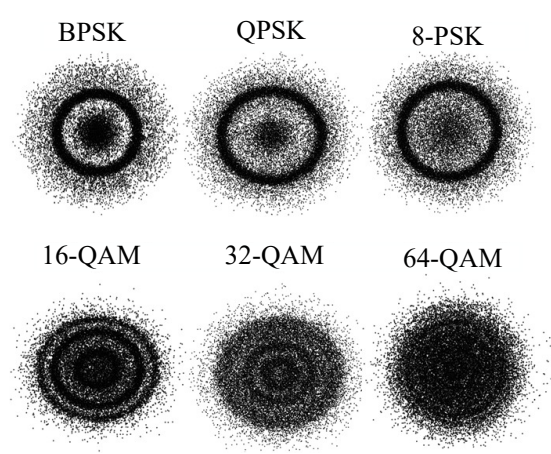

Fig. 2. Sinais BPSK, QPSK, 8-PSK, 16-QAM, 32-QAM e 64-QAM com valor de OSNR igual a $20 \mathrm{~dB} / 0,1 \mathrm{~nm}$, após o bloco $\mathrm{CD}$ compensation.

início a etapa de extração das características, ilustrada na Figura 3. Neste bloco, é realizada a extração do histograma de um sinal 8-PSK com $20 \mathrm{~dB} / 0,1 \mathrm{~nm}$ de OSNR no estado de polarização $\overrightarrow{E_{x}}$. Desta forma, na saída do bloco, obtém-se dois histogramas deste sinal, um em amplitude e fase e outro em amplitude e quadratura com ocorrências, variando entre 0 e 360 . 


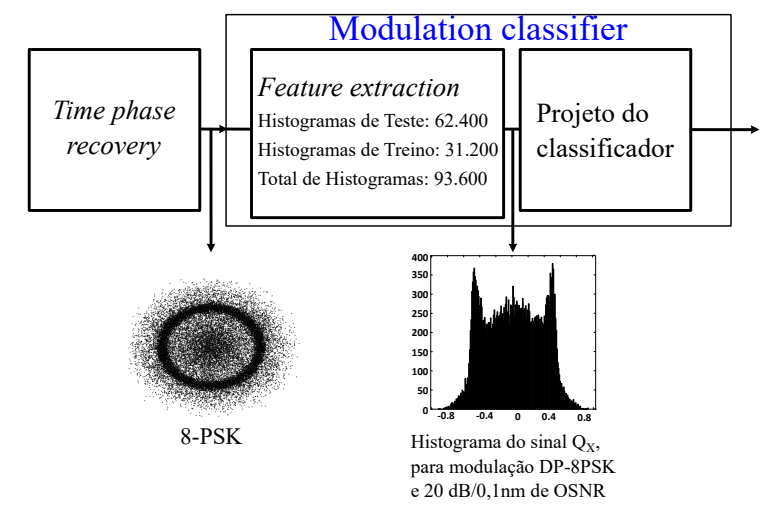

Fig. 3. Extração de características de $\overrightarrow{E_{x}}$ para o sinal em fase de DP-8PSK à 20 e Fig.dB/0, $1 \mathrm{~nm}$.

\section{Algoritmos random forest e árvore de decisão}

Segundo Breiman [24], o algoritmo random forest é definido como um classificador composto por um comitê de $N$ árvores de decisão, as quais realizam uma votação para decidir à qual classe, dentre as previamente treinadas, um determinado conjunto de características (features) pertence. Esse descrição é demonstrada na eq. (1), onde o resultado atribuído a $C$ corresponde a classe com maior frequência no comitê formado pelo voto de cada árvore $h_{n}$ relativo às características $\theta_{n}$ utilizadas por cada árvore, presentes no vetor de entrada $\mathbf{x}$.

$$
C=\left\{h_{n}\left(\mathbf{x}, \theta_{n}\right)\right\}, \operatorname{com} n=\{1, \ldots, N\}
$$

Para comparações, também utilizamos uma variação do popular algoritmo de árvore de decisão C4.5 denominado CART.

\section{E. Algoritmo adaboost}

O adaboost (do inglês, adaptive boosting) é um algoritmo que utiliza um classificador fraco (weak classifier) como entrada e durante o treinamento combina-se, normalmente de maneira ponderada, diversos weak classifiers ligeiramente diferentes. Ao final deste procedimento, obtém-se o classificador (geral), denominado classificador forte (strong classifier) [25]. A eq. (2) representa a saída do classificador forte, onde $C(\mathbf{x})$ é a regra de classificação encontrada ao fim do treino, $\alpha$ são os pesos dos modelos, $T(\mathbf{x})$ representa a resposta de cada weak classifier ao utilizar um vetor de entrada $\mathbf{x}$. A versão do algoritmo adaboost utilizada neste artigo denominase adaboost-SAMME [25].

$$
C(\mathbf{x})=\sum_{m=1}^{M} \alpha_{m} T_{m}(\mathbf{x})
$$

\section{F. Projeto e parâmetros dos classificadores}

Nesta pesquisa, conforme comenta-se acima, foram considerados os algoritmos random forest, árvore de decisão e adaboot. Para avaliação de desempenho destes algoritmos, utiliza-se versões implementadas na biblioteca scikit-learn [26] escrita em linguagem Python. Assim, o conjunto de histogramas para treinamento e os valores dos parâmetros descritos nas Tabelas I e II são utilizados para o projeto dos respectivos classificadores.

\section{G. Métricas, avaliação e resultados}

Para a avaliação dos resultados obtidos durante a etapa de treino e teste realizada, foram utilizadas métricas que
TABELA I

PARÂMETROS DOS CLASSIFICADORES RANDOM FOREST E ÁRVORE DE

\begin{tabular}{|c|c|}
\hline \multicolumn{2}{|l|}{ Parâmetros } \\
\hline Nome & Valor \\
\hline Método de aleatorização das amostras ${ }^{\star}$ & Bootstrap \\
\hline Núm. máximo de características $(F)$ & $\sqrt{F} \approx 14$ \\
\hline Núm. de amostras utilizadas ${ }^{\star}$ & 32.768 \\
\hline Critério para divisão dos ramos & Coeficiente Gini \\
\hline Núm. de amostras para criar um ramo & $\geq 2$ \\
\hline Núm. de amostras para criar uma folha & $\geq 1$ \\
\hline Valor de impureza para criar um ramo & $\overline{\geq} 0$ \\
\hline Valor de impureza para criar uma folha & $\geq 0$ \\
\hline Quantidade máxima de folhas & $\infty$ \\
\hline Profundidade máxima de uma árvore & $\infty$ \\
\hline Cálculo do erro médio & Não utilizado \\
\hline Dicionário com os pesos de cada classe & {$\left[\begin{array}{llll}1 & 1 & \ldots & 1\end{array}\right]$} \\
\hline Quantidade de árvores* & 10 \\
\hline Estratégia para separação dos $\operatorname{ramos}^{\circ}$ & Melhor divisão \\
\hline
\end{tabular}
DECISÃO.

TABELA II

PARÂMETROS DO CLASSIFICADOR ADABOOST.

\begin{tabular}{|l||l|}
\hline \multicolumn{2}{|c|}{ Parâmetros } \\
\hline Nome & Valor \\
\hline \hline Classificador base & Decision tree \\
Quantidade de estimadores & 50 \\
Peso atribuído $(\alpha)$ após cada iteração & 1 \\
Algoritmo adaboost & SAMME.R \\
\hline
\end{tabular}

auxiliassem a avaliação do desempenho dos modelos produzido, para isso, foram utilizadas a acurácia no treino e no teste, o erro absoluto médio, o erro quadrático médio e raiz do erro quadrático médio. Para garantir a generalização dos classificadores obtidos, aplica-se o procedimento de cross validation [26]. Neste, considera-se uma partição disjunta dos sinais para treinamento e teste do classificador, sendo este procedimento $(k$-fold) repetido $k=10$ vezes. As Figuras 4, 5 e 6 contêm as matrizes de confusão dos respectivos algoritmos random forest, adaboost e árvore de decisão.

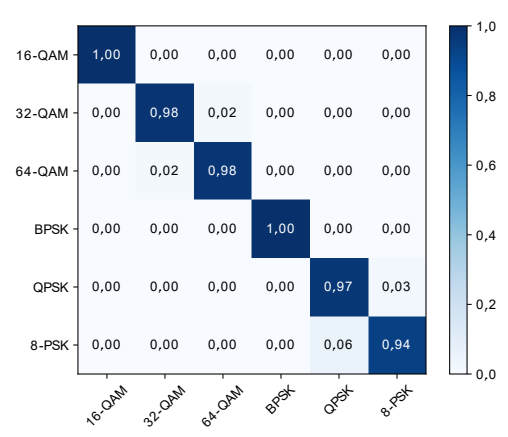

Fig. 4. Matriz de confusão para o algoritmo random forest.

\section{CONCLUSÕES}

Neste trabalho apresentou-se uma pesquisa a respeito da utilização dos algoritmos de classificação random forest, adaboost e árvore de decisão para classificação automática de seis modulações (DP BPSK, DP QPSK, DP 8-PSK, DP 16QAM, DP 32-QAM e DP 64-QAM) em receptores ópticos coerentes flexíveis. A extração de histogramas referentes aos sinais em fase, em amplitude e em quadratura de apenas uma das polarizações, mostrou-se bem promissora, mesmo para os 


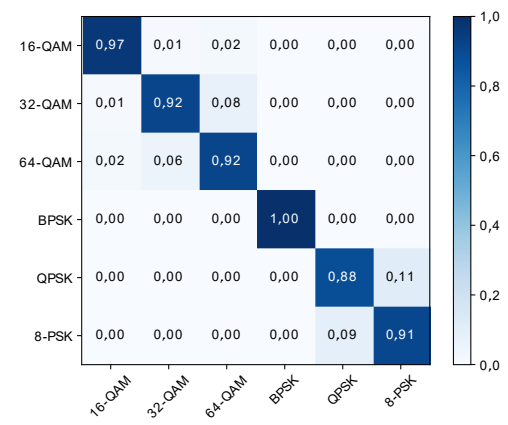

Fig. 5. Matriz de confusão para o algoritmo adaboost.

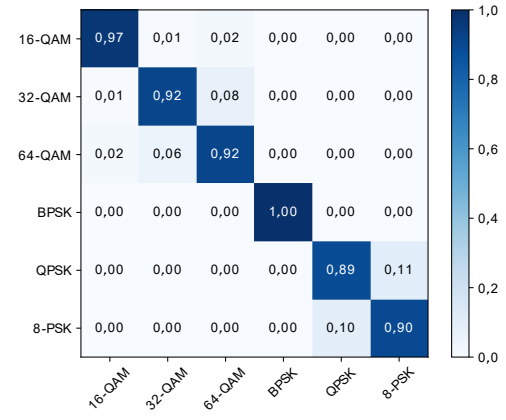

Fig. 6. Matriz de confusão para o algoritmo árvore de decisão.

níveis de ruídos aqui utilizados entre 14 e $20 \mathrm{~dB} / 0,1 \mathrm{~nm}$ de relação sinal ruído. Os resultados obtidos foram equivalentes, com os algoritmos random forest, adaboost, árvore de decisão apresentado as taxas de acerto respectivamente acima de $97 \%$. $\mathrm{O}$ pior resultado foi apresentado pelo algoritmo adaboost com uma taxa e acerto de $88 \%$ para a modulação QPSK. Como trabalhos futuros, pode-se estender a pesquisa para classificações automáticas que utilizam arquiteturas de redes neurais rasas (shalow neural networks) em detrimento às redes profundas, que estão atualmente sendo bastante exploradas. Neste caso, as redes rasas possuem vantagens atrativas como treinamento e execução mais rápidas, além de precisar de um número menor de sinais para treinamento.

\section{Agradecimentos}

Parte dos resultados deste artigo foram financiados por ENVISION Indústria de Produtos Eletrônicos LTDA nos termos da Lei Brasileira Federal No. 8.387/91 (SUFRAMA).

\section{REFERÊNCIAS}

[1] CISCO annual Internet report (2018-2023). https://www.cisco.com/c/en /us/solutions/collateral/executive-perspectives/annual-internet-report/ white-paper-c11-741490.html, 2020

[2] Mamta Agiwal, Abhishek Roy, and Navrati Saxena. Next generation $5 \mathrm{~g}$ wireless networks: A comprehensive survey. IEEE Communications Surveys Tutorials, 18(3):1617-1655, 2016.

[3] G. Fettweis, H. Boche, T. Wiegand, E. Zielinski, and et. al. The tactile Internet. ITU-T Technology Watch Report, page 18, Aug 2014

[4] CISCO Corporation. Internet of things. https://www.cisco.com/c/en/us/ products/collateral/se/internet-of-things/at-a-glance-c45-731471.pdf, 2016.

[5] Khaled B. Letaief, Wei Chen, Yuanming Shi, Jun Zhang, and YingJun Angela Zhang. The roadmap to 6G: AI empowered wireless networks. IEEE Communications Magazine, 57(8):84-90, 2019.

[6] Ranaweera C., Nirmalathas A., Wong E., Lim C., Monti P., Furdek M., Wosinska L., Skubic B., and Machuca C. M. Rethinking of optical transport network design for $5 \mathrm{G} / 6 \mathrm{G}$ mobile communication. IEEE Future Networks Tech Focus, 2021.

[7] L. C. Kimerling, D. Ahn, A. B. Apsel, M. Beals, and et. al. Electronic photonic integrated circuits on the CMOS platform. In Silicon Photonics, volume 6125, pages 6-15. International Society for Optics and Photonics, SPIE, 2006.
[8] Pincemin E., Karaki J., Loussouarn Y., Poignant H., B. C., T. Gilles, and Bidan R. L. Challenges of 40/100Gbps and higher-rate deployments over long-haul transport networks. Optical Fiber Technology, 17(5):335-362, 2011.

[9] Pincemin E., M. Song, J. Karaki, O. Zia-Chahabi, and et. al. Multiband OFDM transmission at 100 Gbps with sub-band optical switching. Journal of Lightwave Technology, 32(12):2202-2219, Jun 2014.

[10] V.E.S. Parahyba, J.D. Reis, S.M. Ranzini, and et. al. Performance against implementation of digital backpropagation for high-speed coherent optical systems. Electronics Letters, 51:1094-1096(2), July 2015.

[11] Maria Ionescu, Domanic Lavery, Adrian Edwards, Eric Sillekens, and et. al. $74.38 \mathrm{~Tb} / \mathrm{s}$ transmission over $6300 \mathrm{~km}$ single mode fibre enabled by $\mathrm{C}+\mathrm{L}$ amplification and geometrically shaped PDM-64QAM. Journal of Lightwave Technology, 38(2):531-537, 2020.

[12] ITU-T Recommendations. G.694.1: Spectral grids for WDM applications: DWDM frequency grid, series G: Transmission system and media, digital systems and networks, Fev. 2012.

[13] Javier Mata, Ignacio de Miguel, Ramón J. Durán, Noemí Merayo, Sandeep Kumar Singh, Admela Jukan, and Mohit Chamania. Artificial intelligence (AI) methods in optical networks: A comprehensive survey. Optical Switching and Networking, 28:43-57, 2018.

[14] L. Schanner. Evaluation of frequency-domain learned digital backpropagation nonlinear compensation for unrepeatered optical links, 2020.

[15] Borges L. N., Costa C., Paula R., Abbade M. L. F., and Aldaya I K-means clustering for mitigation of nonlinear phase noise in digital coherent optical systems using 16-QAM modulation format. In Proc. Simp. Brasileiro de Telecom. e Proc. de Sinais (SBrT), pages 393-397, 2020.

[16] Yan Zhao, Chen Shi, Tao Yang, Jinhao Du, Yuanru Zang, Danshi Wang, Xue Chen, Liqian Wang, and Zhiguo Zhang. Low-complexity and joint modulation format identification and osnr estimation using random forest for flexible coherent receivers. Optics Communications, 457:124698, 2020.

[17] Xiang Lin, Yahia A. Eldemerdash, Octavia A. Dobre, Shu Zhang, and Cheng Li. Modulation classification using received signal's amplitude distribution for coherent receivers. IEEE Photonics Technology Letters, 29(21):1872-1875, Nov 2017.

[18] Jakob Thrane, Jesper Wass, Molly Piels, Julio C. M. Diniz, Rasmus Jones, and Darko Zibar. Machine learning techniques for optical performance monitoring from directly detected pdm-qam signals. Journal of Lightwave Technology, 35(4):868-875, 2017.

[19] Faisal Nadeem Khan, Kangping Zhong, Waled Hussein Al-Arashi, Changyuan $\mathrm{Yu}$, Chao Lu, and Alan Pak Tao Lau. Modulation format identification in coherent receivers using deep machine learning. IEEE Photonics Technology Letters, 28(17):1886-1889, 2016.

[20] D. Amoedo, Wheidima C. Melo, L. C. Cordeiro, Eddie B. L. Filho, W. Junior, and C. Carvalho. Classificação automática de modulações mono e multiportadoras utilizando método de extração de características e classificadores SVM. In Proc. Simp. Brasileiro de Telecom. e Proc. de Sinais (SBrT), 2017.

[21] Valadão M. D. M., Pereira A. M. C., Amoedo D. A., Filho E. B. L. Carvalho C. B., Costa A. L. A., Cordeiro L. C., and Júnior W. S. S. Classificação automática de modulações utilizando redes neurais artificiais com regularização bayesiana e algoritmo de retropropagação de levenberg-marquardt. In Proc. Simp. Brasileiro de Telecom. e Proc. de Sinais (SBrT), pages 393-397, 2019.

[22] Francesco Musumeci, Cristina Rottondi, Avishek Nag, Irene Macaluso, Darko Zibar, Marco Ruffini, and Massimo Tornatore. An overview on application of machine learning techniques in optical networks. IEEE Communications Surveys Tutorials, 21(2):1383-1408, 2019.

[23] Qianwu Zhang, Hai Zhou, Yuntong Jiang, Bingyao Cao, Yingchun Li, Yingxiong Song, Jian Chen, Junjie Zhang, and Min Wang. A simple joint modulation format identification and OSNR monitoring scheme for IMDD OOFDM transceivers using k-nearest neighbor algorithm. Applied Sciences, 9(18), 2019.

[24] Leo Breiman. Random forests. Machine learning, 45(1):5-32, 2001.

[25] Trevor Hastie, Saharon Rosset, Ji Zhu, and Hui Zou. Multi-class adaboost. Statistics and its Interface, 2(3):349-360, 2009

[26] F. Pedregosa, G. Varoquaux, A. Gramfort, V. Michel, B. Thirion, O. Grisel, M. Blondel, P. Prettenhofer, R. Weiss, V. Dubourg, J. Vanderplas, A. Passos, D. Cournapeau, M. Brucher, M. Perrot, and E. Duchesnay. Scikit-learn: Machine learning in Python. Journal of Machine Learning Research, 12:2825-2830, 2011. 